\section{BCA Symposium Considers Diffraction from Nearly Perfect Crystals}

The British Crystallography Association (BCA) and Institute of Physics Physical Crystallography Group organized a symposium on "Diffraction from Nearly Perfect Crystals" during the Solid State Physics Conference in Bristol, December 16-18, 1987. The symposium comprised three sessions, all on slightly different themes and all proving very successful. Mary Halliwell (British Telecom Research Laboratories) organized the scientific program and Andrew Lang arranged the local requirements for the sessions.

The first session was based on studies on Si. Joachin Schneider (Hahn-Meitner Institute) presented his work on $\gamma$-ray diffraction. The typical wavelength is $0.039 \AA$ and a typical sample will be $1 \mathrm{~cm}$ thick and 10 $\mathrm{cm}$ in diameter. The advantages of this are the low absorption, no anomalous dispersion and the extinction is easy to handle, giving rise to an indefinite number of Pendellösung fringes allowing accurate measures of thicknesses and structure factors. The absence of multiple scattering is checked by the complementary nature of the transmitted and diffracted rocking curves. Schneider determined the strain fields arising from $\mathrm{SiO}_{2}$ precipitates in $\mathrm{Si}$ samples by the slope in the average reflectivity vs. thickness line by tilting the crystal. The strain fields from these clusters appear to extend over a few thousand angstroms, and are not fully understood. Peter Halfpenny (Hirst R.C.) gave a good account of topography on processed $\mathrm{Si}$ and gave examples of defects caused by mechanical, elastic and thermomechanical stresses. This he related to the original material and the processing.

Keith Bowen et al. (Warwick University) used double crystal rocking curves to assess residual saw damage in Si wafers. The work showed that low energy ion beam milling can result in an acceptable surface; no improvement was found by an initial etch of the surface or by pursuing the ion beam milling beyond a certain depth removal. Mary Halliwell et al. (British Telecom) presented studies on $\mathrm{Si} / \mathrm{SiGe}$ superlattice structures, including the comparison of the modulation period determined by $x$-ray diffraction, Raman, and TEM. The first two agreed well, but the TEM results differed for low strain levels because of the sampling volume to dislocation ratio, although it did show the dislocation distribution from the relaxation process.
In a colorful talk, Andrew Lang (Bristol) threw much light on the study of defects in diamond. He summarized the optical classification of these materials in relation to nitrogen defects and described the effects of natural and artificial radiation on diamond, indicating their potential as lasing materials. Mixed habit growth was studied by "spike" $x$-ray topography (on the SRS) and by cathodoluminescence. This showed the formation of platelet-like "laths," which was verified by TEM. In a second example, the formation of "negative crystals" or "voidites" due to the absence of a platelet was found by TEM. Very recent EELS work suggests that these consist of a high pressure form of fcc nitrogen. John Sherwood (Strathclyde) discussed the growth of high purity organic crystals for use in optoelectronic materials. Such crystals have high damage thresholds and so need to be optically "perfect." In describing growth methods such as the Bridgman and Czochralski techniques, he indicated how thermally induced dislocations during growth could be seen by SRS Laue topography. The nature of each growth technique produces defects peculiar to that method. Aspects of solution growth methods were also covered highlighting the importance of the seed crystal growth quality. Despite the care needed in cutting and polishing, remarkable examples were exhibited.

Brian Tanner (Durham) described the use of both $x$-ray and neutron topography to investigate the spin re-orientation transition in hematite. This transition occurs at different temperatures in different domains in the crystal. These "finger-like" domains were found to correlate with growth bands in the crystal and it was suggested that the incorporation of $V$ from the growth flux into the lattice may be responsible for the observed effects. Peter Hatton (Edinburgh) set out to reveal a few of the remaining surprises from the cubictetragonal phase transition in $\mathrm{SrTiO}_{3}$. High resolution $x$-ray scattering measurements of the width of the central point scattering has revealed a second critical length scale of $10^{4} \AA$ just above $T_{c}$. A remarkable hysteresis effect was found wherein cycling through $T_{c}$ reduces the linewidth (i.e., increase of the correlation length) with this processing being "remembered" by the sample for several months.

The third session of the Diffraction Symposium was devoted to three invited papers on advanced semiconductor structures. Colin Humphreys (Liverpool) gave a wide ranging review of high resolution electron microscopy, discussing its ap- plications to GaAs/GaAlAs structures, $\mathrm{SiGe}$ alloys on $\mathrm{Si}$, and $\mathrm{GaAs}$ on $\mathrm{Si}$. The HREM offers much more localized information than x-ray diffraction and is being widely used for studying interface problems. As for x-ray diffraction, the unit cell symmetry within each layer of a multilayer structure can be determined, but in the case of the ultrathin sections used for electron microscopy the symmetry will be modified by the sample preparation procedure. Electron micrographs illustrating how dislocations propagated within strained layer structures were shown.

Paul Fewster (Philips Research Laboratories) described $x$-ray methods of characterizing multiquantum well (MQW) structures (i.e., alternating layers of GaAs and GaAlAs). By computer-assisted matching techniques he was able to obtain remarkable agreement between experimental and theoretical curves and hence detect very thin interfacial layers of graded composition. Roger Cowley (Edinburgh), more widely known for his expertise in neutron diffraction, then described the advantages of the $x$-ray triple crystal diffractometer for studying the 2-D scattering occurring around a reciprocal lattice point. The Edinburgh diffractometer was originally built for phase change investigations but has more recently been applied to semiconductor surface structures. Work was described on the characterization of thin $(<100 \AA)$ amorphous oxide layers and heteroepitaxial single quantum well structures.

The Surfaces and Interfaces of Advanced Semiconductors Symposium, which ran immediately following the Diffraction Studies Symposium, included some additional diffraction work, notably two invited talks covering 2-D $x$-ray diffraction on silicides and MBE grown layers (in situ) performed using synchrotron radiation, and a new method of studying MQWs using convergent beam microscopy on plan view samples developed at Bristol University.

A plenary talk on the use of $x$-ray diffraction methods for the study of materials was given by Michael Hart (Manchester) prior to the third session. He covered many applications of diffraction and spectroscopic techniques with special emphasis on new methods emerging, for example grazing incidence scattering for studying surfaces.

Craig D. Adam

Paul F. Fewster Mary A.G. Halliwell 